\title{
STUDIES ON INBREEDING
}

III. THE EFFECTS OF INBREEDING, WITH SELECTION, ON THE SEX RATIO OF THE ALBINO RAT

HELEN DEAN KING

The Wistar Institute of Anatomy and Biology

ONE FIGURE

During the latter part of the nineteenth century it was generally believed that sex in man and in various animals is determined mainly by the amount of nourishment that the embryos receive; well nourished embryos were supposed to become females; those that were poorly nourished were assumed to develop into males. A considerable amount of evidence in favor of this view was collected by Düsing ('83, '84, '86), who maintained, furthermore, that close inbreeding interferes with embryonic nutrition, by lessening the vitality of the mother, and so produces a great excess of male young.

In the literature of the succeeding twenty years that deals with the subject of sex determination, Düsing's statement regarding the effect of inbreeding on the sex ratio was widely quoted and generally credited. Those who challenged the truth of the assertion were, in the main, advocates of the ancient theory, generally ascribed to Hippocrates (460-377 B.C.), that sex is determined in the ovary; eggs from the right ovary producing males and those from the left ovary developing into females. During this period three series of experiments were made that give data regarding the sex-proportions in a closely inbred stock. Huth ('87) inbred rabbits, brother and sister, for six generations and found a relatively low sex ratio (78.8 $\sigma^{7}: 100$ \%) among the ninety young in which the sex was ascertained; Copeman and Parsons ('04) obtained a similar result in their inbreeding experiments with mice. Schultze ('03) concluded that inbreeding 
has no pronounced tendency to produce an excess of male young, although he found a high sex ratio (110.9 $\left.\sigma^{\pi}: 100 \%\right)$ among 135 mice that were the offspring of brother and sister matings. The question as to whether inbreeding does or does not alter the sex ratio was not satisfactorily answered by any of these experiments, for in each case the number of animals used was small, and there was, apparently, no selection of the best stock for breeding or any way of checking the results. Moreover, none of these investigations were continued long enough to give evidence that could be considered as conclusive.

The effects of inbreeding on the sex ratio seemed to me to be a problem of sufficient importance to warrant a careful and prolonged investigation. For if it were possible to swing the sex ratio of any animal in a definite direction by factors that could be controlled, one might hope to gain valuable information regarding the nature of sex-a problem that has been a favorite subject of speculation for many centuries and one that modern methods of research have not, as yet, satisfactorily solved.

\section{MATERIAL, METHOD, AND SCOPE OF THE INVESTIGATION}

The albino rat (Mus norvegicus albinus) was the animal used in this investigation, which was begun in 1909 . Details regarding the manner in which the experiments were conducted were given in the first paper of this series (King, '18), but it has seemed advisable to repeat them here in order to give a clear understanding of the way in which the problem has been approached.

The basis of the inbred strain was a litter of four albino rats, two males and two females, taken from the general colony of these animals maintained at The Wistar Institute of Anatomy and Biology in Philadelphia. The litter was selected for the purpose in view solely because of its size, not because of the ancestry or the vigor of the animals. One of the two females in the litter was called ' $A$ ', and her descendants form the A series of inbreds; the other female was called ' $B$ ', and her descendants are the $B$ series of inbreds.

Since the mating of brother and sister from the same litter is the closest form of inbreeding possible in mammals, such matings 
would be expected to be more potent than any other kind in producing an alteration in the sex ratio. In these experiments, therefore, brother and sister matings only were used to obtain strictly inbred litters from which all females used for breeding were taken. The plan of breeding that was followed through the first twenty-five generations of these animals was this: Females $A$ and $B$, as well as all of the females in their respective lines that were subsequently used for breeding, were paired twice with a litter brother and then twice with an unrelated male taken from the stock colony. Sex records for the first two litters produced by any group of females might be expected to show whether inbreeding had any effect on the sex ratio; sex data for the third and for the fourth litters cast by these same females would, it was hoped, indicate whether the male or the female was responsible for the alteration, if any, in the sex ratio. For convenience the litters obtained from the mating of inbred females with stock males are here designated as 'half-inbred' litters; no animals from such litters have ever been reared.

Emphasis should be placed on the fact that, with few exceptions, the sex data given in this paper were obtained by examining the litters very soon after their birth. The sexes can readily be distinguished at this time, as Jackson ('12) has shown, and if accurate sex data are wanted it is imperative that they be taken as soon as possible, since the young that are stillborn, or those that die soon after birth, are usually eaten by the mother within a few hours.

In order to keep track of a large series of animals it was necessary to find some way in which the pedigree of any particular individual could be told by a glance at the record card. The scheme of marking devised, which is outlined below, has proved to be very convenient and also most satisfactory for the filing of permanent records. The letter A or B is used to show from which of the two females, A or B, the animal was descended, and thus places the individual in its proper series. The serial letter is preceded in all cases by a number which signifies the generation to which the animal belonged. An index number, 2, 3, or 4, following the serial letter shows in which of the mother's litters 
the animal was born; if no index number is used the rat was a member of its mother's first litter. The subseript following the serial letter is the number which serves to distinguish each particular rat from the other rats belonging to the same generation and litter group. When it is desired to indicate the sex of the individual its number is inclosed by the sex symbol. An illustration will, perhaps, render the scheme clearer.

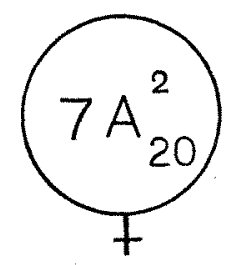

This symbol denotes a female rat belonging in the seventh generation of the $A$ series of inbreds. She was a member of the second litter cast by her mother, and her individual number in the series of rats belonging to the second litters of the seventh generation was twenty.

In the early generations of both inbred series the animals suffered severely from malnutrition which produced a marked effect on their growth, fertility, and longevity, as previous papers in this series have shown (King, '18, '18 a). During this period a considerable proportion of the individuals were sterile, and it was not possible to select animals for breeding; any rats that would breed at all were used to continue the strain. Nutritive conditions were improved at the time that the rats of the fourth inbred generation were approaching maturity, and a decided improvement in the condition of the animals was noted in a very short time: they gained rapidly in weight, the litters cast became larger and sterility almost disappeared. At this stage of the investigation it became possible to attempt to alter the sex ratio by selection within the inbred strain. From the seventh generation on, every female in the A series of inbreds that was used for breeding was taken from a litter that contained an excess of males; breeding females in the $B$ series of inbreds were all taken from litters containing an excess of females. The plan 
of pairing a female twice with a litter brother and then twice with an unrelated stock male was continued through the first twenty-five generations of both inbred series.

In each series litters having the desired sex ratio were reared as possible breeding stock only when the young were of large size and lusty at birth; all other litters were discarded regardless of their sex ratio. At the time that the animals became sexually mature the largest and most vigorous pairs were the ones taken to continue the strain. Selection of breeding stock, it will be noted, was based primarily on the sex ratio in the litters, not on the size or on the vigor of the young. This means that the animals in one generation that became the progenitors of the succeeding generation were selected because of their parents, tendency to produce young of a certain sex. A pair of rats that produced two litters, each of which had the desired sex ratio, was considered as having an unusually strong tendency to produce unisexual young; individuals from each of these litters were used for breeding when possible. The basis of the selection, therefore, was along the line in which Pearl ('12, '12 a, '17) has obtained such marked success in increasing egg production in poultry, i.e., according to the ability of the parents to transmit to the offspring the quality desired.

In the early part of this investigation the number of breeding females was, of necessity, small, but in the later generations about twenty females in each series were used for breeding, so that at least 1000 rats were obtained in each generation of the inbred strain. Sex records for the first twenty-five generations are given in the present paper; the data comprise 3408 litters containing 25,452 individuals.

\section{THE NORMAL SEX RATIO IN THE ALBINO RAT}

The normal sex ratio in any species can properly be determined only by obtaining the sex data for the total number of offspring produced by many females during the entire period of their reproductive activity. Unfortunately, no such series of data for the albino rat have been recorded, and only two sets of observations regarding the normal sex ratio in this animal have, 
as yet, appeared. Cuénot ('99) examined thirty litters of albino rats, containing 255 young, and found a sex ratio of $105.6 \mathrm{o}^{7}$ : 100 o ; data for 1089 litters of stock Albinos, collected by King and Stotsenburg ('15), gave a sex ratio of $107.5 \sigma^{7}: 100$ ㅇ․ Neither of these determinations seemed to furnish a proper standard for comparison with the sex ratios obtained in the inbred strain, even though they differed by less than two points. The number of individuals examined by Cuénot was too small to give results of much statistical value. The sex ratio given by King and Stotsenburg was based on the findings for a relatively large number of animals, but the litters recorded were, for the most part, cast by females that had not reached the height of their reproductive activity. The sex ratio among the offspring of young females could not justly be taken as a norm for the Albino strain in general, since it has been shown that in the albino rat the sex of the young seemingly depends, to a certain extent, on the age of the mother (King, ' 16 a).

In order to ascertain the normal proportion of the sexes in the strain of Albinos from which the inbred animals were taken, I obtained the complete breeding history of a considerable number of stock females during the past four years. As all of these individuals were reared under the same environmental conditions as the inbred rats, the sex ratio among their young would seem to be a suitable standard by which to judge the sex ratios found in various generations of the inbred animals. To make the ratios more strictly comparable, the data for only the first four litters of the stock series were used in computing the sex ratio which was to serve as the norm. These data, arranged by litter groups, are shown in table 1.

TABLE 1

Showing the sex ratios in the first four litters of a series of stock albino rats

\begin{tabular}{|c|c|c|c|c|c|}
\hline LTTER SERIDS & NUMBER LITTERS & $\begin{array}{l}\text { NUMBER } \\
\text { INDIVIDEALS }\end{array}$ & MALES & FEMALES & $\begin{array}{l}\text { NUMBER MALES } \\
\text { TO } 100 \text { FEMALES }\end{array}$ \\
\hline 1 & 116 & 717 & 385 & 332 & 115.9 \\
\hline 2 & 116 & 843 & 426 & 417 & 102.2 \\
\hline 3 & 103 & 671 & 328 & 343 & 95.6 \\
\hline \multirow[t]{2}{*}{4} & 89 & 587 & 302 & 285 & 105.9 \\
\hline & 424 & 2818 & 1441 & 1377 & 104.6 \\
\hline
\end{tabular}


Table 1 shows that there was a relatively large excess of males in the first litters cast by this series of stock females $\left(115.90^{7}\right.$ : 100 o), and that in succeeding litters the sex ratio tended to fall considerably. A similar change in the sex ratio of successive litters of mice was noted by Copeman and Parsons ('04), and was found also by King and Stotsenburg ('15; table 7) in a series of stock albino rats. Large groups of statistics for human births, as summarized by Ahlfeld ('76), by Düsing ('83, '84), by Punnet ('03), and by Newcomb ('04), all show that the sex ratio is very high among the first children of young mothers and then tends to fall with succeeding births until the mother is about thirty years old. Whether a similar change in the sex ratio is characteristic of other mammals has not been determined as yet.

Among the 2818 individuals comprised in this series of stock litters there were 104.6 males to each 100 females. A sex ratio of $1050^{x}: 100$ o was, therefore, taken as the norm by which to judge the sex ratios obtained in the various groups of inbred rats. This sex ratio, it will be noted, is very close to that given by Cuénot, and is lower, by over two points, than the sex ratio found in the large group of stock Albinos born in The Wistar Institute colony during the years 1911-1914 (King and Stotsenburg, '15).

\section{THE SEX RATIO IN INBRED LITTERS OF ALBINO RATS}

The A series of inbred rats may be designated as the 'male line,' since after the sixth generation all of the breeding females in this series were taken from litters that contained an excess of males. Table 2 gives, by litter groups, the sex data for the 13,116 individuals obtained in the first twenty-five generations of this series.

Table 2 is inserted chiefly for reference, and a detailed analysis of the data, as given, will not be attempted. The summary of the data for the various litter groups shows that the sex ratio for the first litters produced was much higher than that for the second, third, and fourth litters. A similar change in the sex ratio was noted in the litter series of stock animals given in table 1.

The B series of inbreds is called the 'female line,' since, after the sixth generation, all breeding females in this series came from 


\begin{tabular}{|c|c|c|c|c|}
\hline \multirow{2}{*}{ 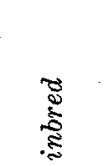 } & \multirow{2}{*}{ 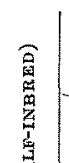 } & 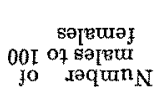 & 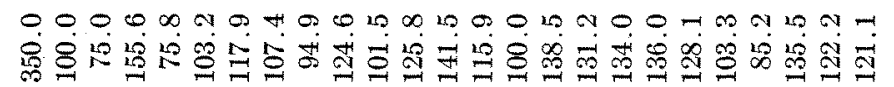 & 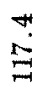 \\
\hline & & 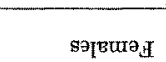 & 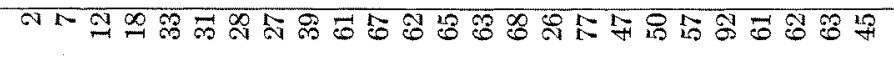 & $\stackrel{8}{8}$ \\
\hline s & 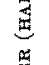 & 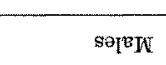 & "- - & 兽 \\
\hline 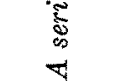 & 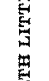 & 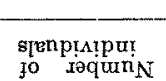 & ○ & 赵 \\
\hline 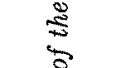 & 宽 & 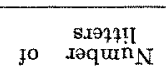 & 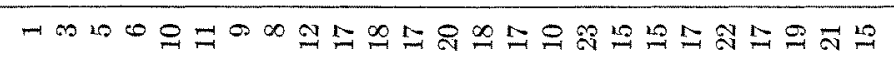 & 总 \\
\hline 产 & $\widehat{\hat{\theta}}$ & 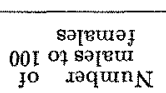 & 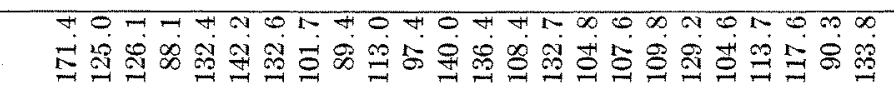 & $\stackrel{0}{=}$ \\
\hline ङ્ँّ & 产 & вәтниэ & 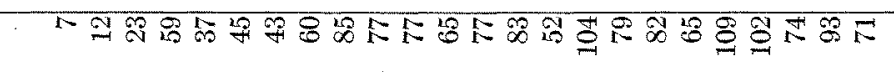 & $\vec{\infty}$ \\
\hline 苋 & 迸 & seren & 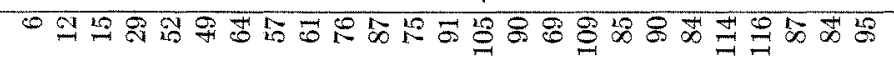 & $\underset{\mathscr{D}}{\mathbb{\infty}}$ \\
\hline 离 & 案 & 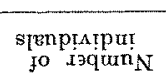 & 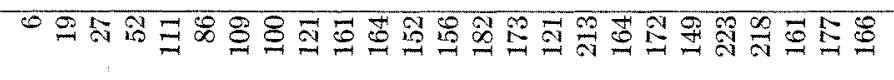 & 要 \\
\hline 要 & 珰 & 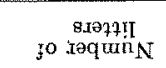 & - & $\stackrel{\infty}{\stackrel{q}{q}}$ \\
\hline 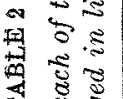 & & 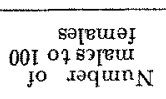 & 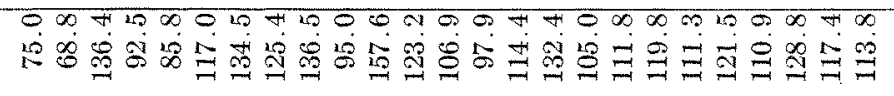 & $\begin{array}{l}P \\
\dot{U}\end{array}$ \\
\hline 离 & 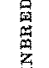 & 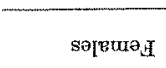 & 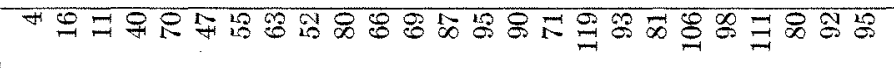 & 官 \\
\hline 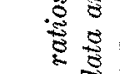 & 爱 & so[B $\mathrm{K}$ & 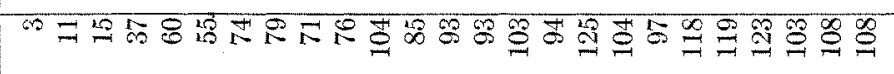 & 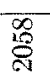 \\
\hline 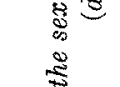 & 望 & $\begin{array}{l}\text { STenptapup } \\
\text { jo dəquni }\end{array}$ & 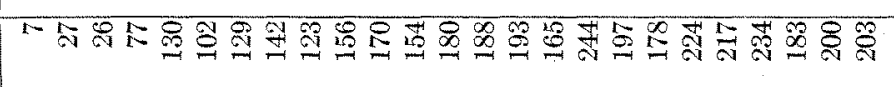 & 要 \\
\hline 丞 & & jo $\operatorname{saquan}_{\mathrm{N}}$ & - & $\mathscr{B}$ \\
\hline 胥 & & 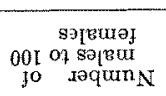 & 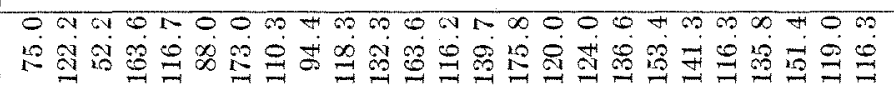 & $\stackrel{8}{\$}$ \\
\hline 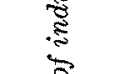 & 憅 & sәгенх & サの & 量 \\
\hline હ & 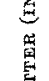 & รәГв & יニ 웡 & $\stackrel{8}{\stackrel{8}{\circ}}$ \\
\hline 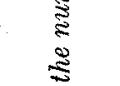 & 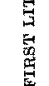 & $\begin{array}{l}\text { slenpratp } \\
\text {-u! jo Iəquinn }\end{array}$ & " & 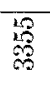 \\
\hline · & & 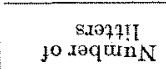 & - & $\stackrel{12}{9}$ \\
\hline & & 舀总 & 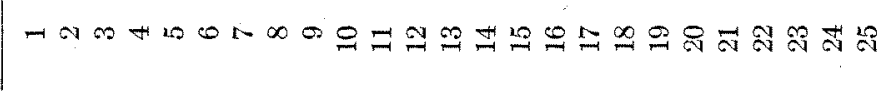 & \\
\hline
\end{tabular}


litters containing an excess of females. Reference data showing the proportion of males and females produced in the various generations of this series are given, by litter groups, in table 3 . The data comprise a total of 1656 litters containing 12,336 individuals.

The summary for each of the four litter groups of the B series (table 3) shows that the sex ratio was at its lowest point in the first litter group, and then tended to rise in each of the subsequent groups. This is a reversed relation of the sex ratios to that shown in the litters of the stock controls (table 1) and in the litter groups of the A series (table 2), and would seem to indicate that some agency, other than environment or the age of the mother, had influenced the relative proportion of the sexes in this series of animals.

In order to compare the sex ratios in the litters sired by inbred males with the sex ratios in the litters sired by stock males, the sex records for the first and second litters produced in each generation of the two series were combined, as were also the records for the third and fourth litters. Table 4 shows the combined data for the litter groups of the A series; table 5 shows similar data for the litter groups of the B series.

Reference to the data given in table 4 and in table 5 will be made later.

To facilitate an analysis of the results obtained in the A series of inbreds, the data, as shown in table 4, were combined in generation groups (table 6). This grouping of the data was purely arbitrary. It seemed useless to compare such large series of records generation by generation, or even to combine the records for two succeeding generations. Since after the sixth generation the selection of breeding animals was made according to a definite plan, it would seem that, logically, the data for the first seven generations should form one group. Such a group, however, was too large for the purpose of ascertaining whether selection produced a varying effect in different generations. It was finally decided to make a total of eight groups, each of which, except the first, should contain the data for three generations. Because of the small number of individuals, records for the first four generations were combined in one group. 


\begin{tabular}{|c|c|c|c|c|}
\hline 8 & 䰹 & 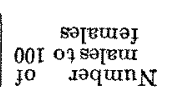 & 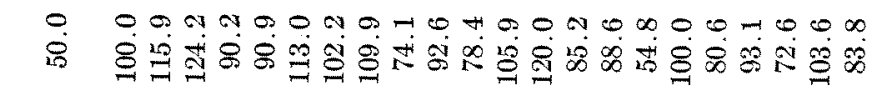 & $\stackrel{\leftrightarrow}{\mathscr{g}}$ \\
\hline .气ूँ & 焉 & 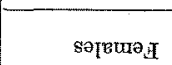 & 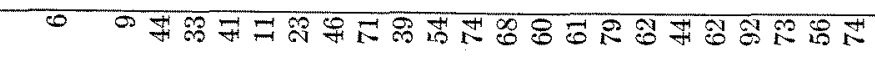 & 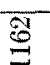 \\
\hline 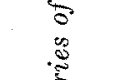 & 起 & sәjex & 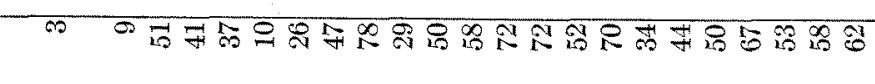 & 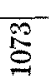 \\
\hline$\infty$ & 蓄 & 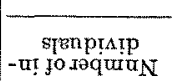 & 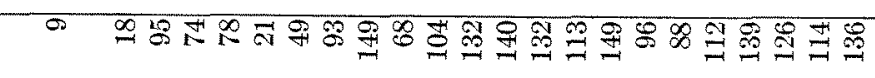 & 莡 \\
\hline 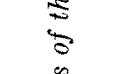 & 窟 & $\sin _{1}$ & 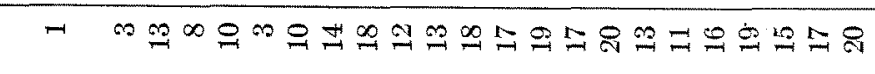 & 今. \\
\hline 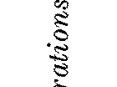 & $\widehat{a}$ & 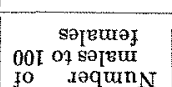 & 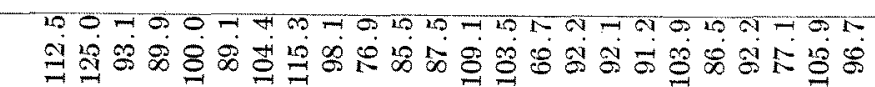 & $\frac{m}{\sigma}$ \\
\hline 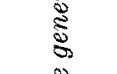 & 爱 & sә[вu保 & 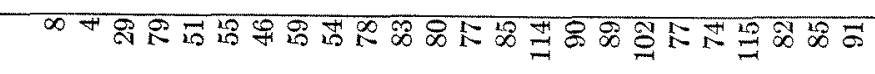 & $\stackrel{5}{S}$ \\
\hline 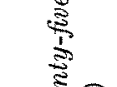 & 道 & se[r'W & 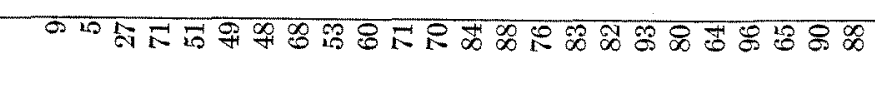 & 承 \\
\hline 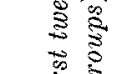 & 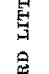 & 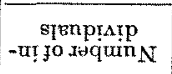 & 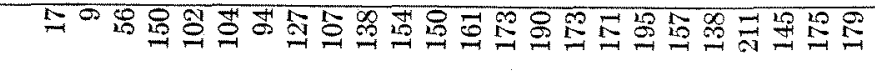 & 赵 \\
\hline 吾弯 & 蟫 & 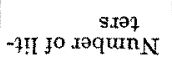 & ง ศト⿻ & $F$ \\
\hline 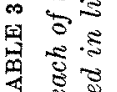 & & 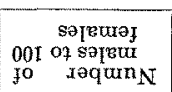 & 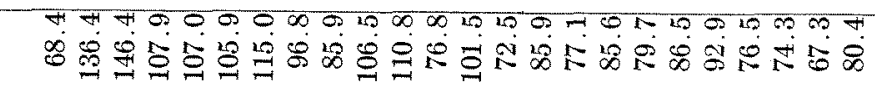 & $\prod_{\infty}^{\infty}$ \\
\hline 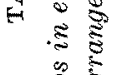 & 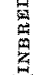 & 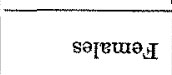 & 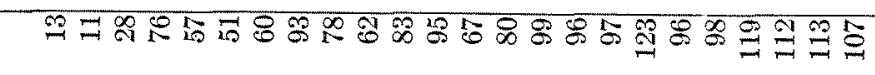 & $\vec{g}$ \\
\hline 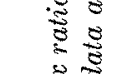 & 善 & selघ & 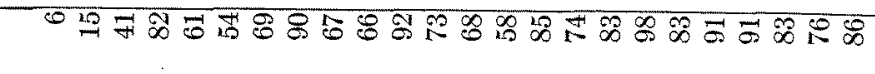 & $\stackrel{\mathscr{B}}{0}$ \\
\hline$\stackrel{3}{\Xi}$ & 至 & 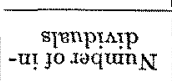 & 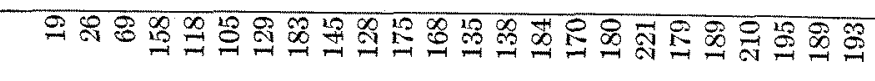 & 悉 \\
\hline 8 & & 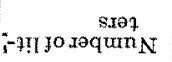 & ㄴ. & ڤै \\
\hline 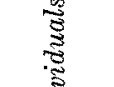 & & 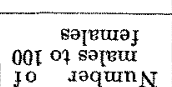 & 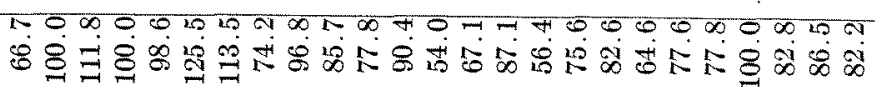 & $\stackrel{\infty}{\infty}$ \\
\hline . & 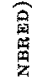 & sә[вura & ம & 营 \\
\hline$\stackrel{\Xi}{\circledR}$ & 通 & sərb]N & 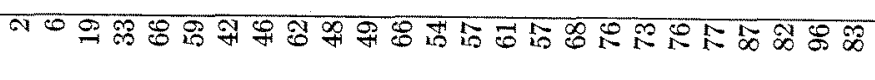 & $\underset{\exists}{\stackrel{3}{\sharp}}$ \\
\hline 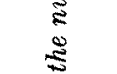 & 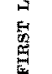 & $\begin{array}{l}\text { signprayp } \\
\text {-u! joxəquin }\end{array}$ & 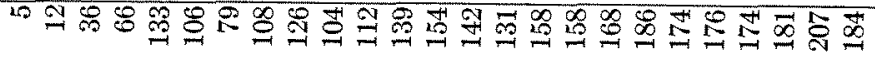 & 공 \\
\hline ·旅 & & 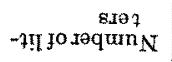 & 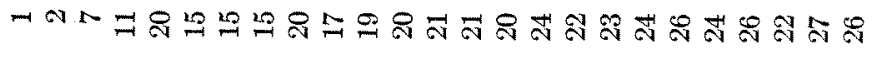 & $\stackrel{\infty}{*}$ \\
\hline & & 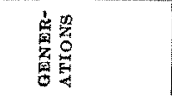 & 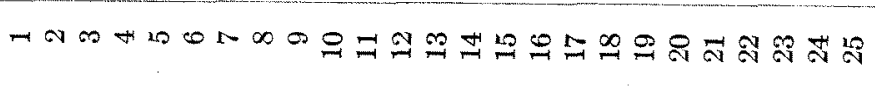 & \\
\hline
\end{tabular}


TABLE 4

Showing the sex ratios in the inbred and in the half-inbred litters produced in each of the first twenty-five generations of the $A$ series of inbreds

\begin{tabular}{|c|c|c|c|c|c|c|c|c|c|c|c|c|c|c|c|}
\hline \multirow[b]{2}{*}{ 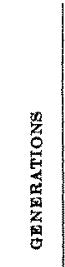 } & \multicolumn{5}{|c|}{$\begin{array}{c}\text { YNBRED } \\
\text { (FIRST AND SECOND LITTERS) }\end{array}$} & \multicolumn{5}{|c|}{$\begin{aligned} \text { HAI } \\
\text { (THIRD AND }\end{aligned}$} & \multicolumn{5}{|c|}{ SUMMARY OF ALL LITTERS } \\
\hline & 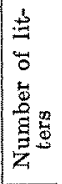 & 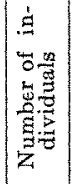 & 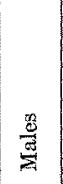 & 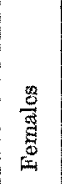 & 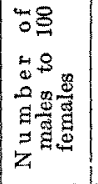 & 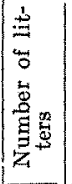 & 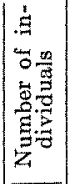 & 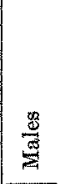 & 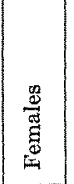 & 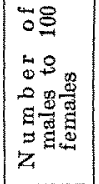 & 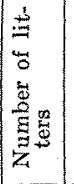 & 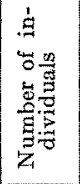 & 总 & 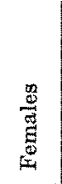 & 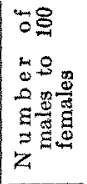 \\
\hline 1 & 2 & 14 & 6 & & 75.0 & & 15 & 13 & 2 & 6 & & 29 & 19 & 10 & \\
\hline 2 & 7 & 47 & 22 & 25 & & & 33 & 19 & 14 & & 13 & 80 & 41 & 39 & \\
\hline 3 & 12 & 61 & 27. & 34 & 79.1 & 10 & 48 & 24 & 24 & 0.0 & 22 & 109 & 51 & 50 & \\
\hline 4 & 25 & $\mid 135$ & 73 & 62 & 117.4 & 14 & 98 & 57 & 41 & 39.0 & 39 & 233 & 130 & 103 & 126. \\
\hline 5 & 36 & 247 & 123 & 124 & & 25 & 169 & & & & & & & & \\
\hline 6 & 30 & & & & 1 & & 149 & & 8. & 1 & & & & & \\
\hline 7 & 32 & 230 & 138 & 92 & & 64 & 170 & & 73 & & & & & & \\
\hline 8 & 34 & 264 & 14 & $1:$ & & & 15 & & 70 & 1 & & & & & \\
\hline 9 & 34 & 2 & 12 & & & 8 & 197 & 98 & 99 & 00 & 2 & 4 & & & \\
\hline 10 & 40 & 2 & & & & & 298 & 152 & 146 & & 7 & 58 & & & \\
\hline 11 & & & & & & & 299 & & & & 80 & 61 & & 2 & \\
\hline & & & & & & & 26 & & & & & & & & \\
\hline 1 & 4 & 340 & & & & & 31 & & 30 & & & & & & \\
\hline 1 & $4:$ & 327 & & & & & & & 10 & & & & & & \\
\hline 1 & 46 & 364 & & & & & & & 1) & & & & & & \\
\hline 16 & 42 & 30 & & & & & 18 & & & & & & & & \\
\hline 1 & & 4 & & & & & 11 & 10 & 181 & 3.0 & 102 & 84 & 51 & & \\
\hline 1 & & 36 & 20 & & & 34 & 27 & & & & & & & & \\
\hline & & 32 & & & & & 29 & & & & & & & & \\
\hline & & 4 & & & & & 2 & & & & & & & & \\
\hline & & & & & & & & & & & 102 & & & & \\
\hline 2 & & 4 & 24 & 19 & & & & & 33 & & 95 & & & & \\
\hline 2 & 5 & 369 & 215 & 18 & & & 30 & & & & 91 & 672 & & & \\
\hline & 5 & 384 & 208 & 17 & & 43 & 317 & 161 & 156 & & 4 & 701 & 369 & 332 & \\
\hline & w & 376 & 201 & 175 & 114.9 & 00 & 270 & 154 & 116 & 132.8 & 90 & 646 & 355 & 291 & 122.0 \\
\hline & & & & & & & & & & & & & & & \\
\hline
\end{tabular}

As the number of individuals in each of the first seven generations of the A series was comparatively small, it is not surprising that the sex ratios in the inbred and in the half-bred groups of litters should show a wide range of variation (table 4). When the records for these generations were combined, as shown in table 6 , it was found that the 144 inbred litters had a sex ratio of $110.40^{7}$ : 
TABLE 5

Showing the sex ratios in the inbred and in the half-inbred litters produced in each of the first twenty-fwe generations of the $B$ series of inbred

\begin{tabular}{|c|c|c|c|c|c|c|c|c|c|c|c|c|c|c|c|}
\hline \multirow[b]{2}{*}{ 齐 } & \multicolumn{5}{|c|}{$\begin{array}{c}\text { INBED } \\
\text { (FIRBT AND SECOND LMTTERB) }\end{array}$} & \multicolumn{5}{|c|}{$\begin{array}{c}\text { HALF-INBRED } \\
\text { (SECND AND THIRD LITTERS) }\end{array}$} & \multicolumn{5}{|c|}{ SUMMARY OF A } \\
\hline & 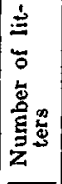 & 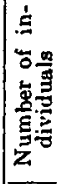 & 离 & 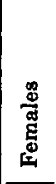 & 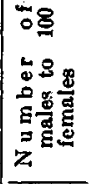 & 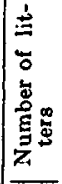 & 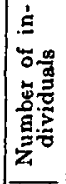 & 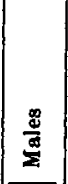 & 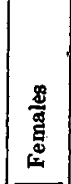 & 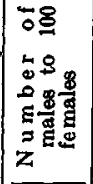 & 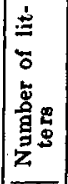 & 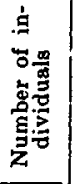 & 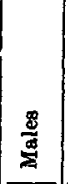 & 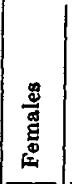 & 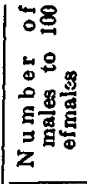 \\
\hline 1 & 1 & 5 & & & & & & & & & & b & 2 & & \\
\hline 2 & 4 & 31 & 12 & 19 & 63.2 & 3 & 26 & 12 & 14 & 85.7 & 7 & 57 & 24 & 33 & \\
\hline 3 & 12 & 62 & & 28 & 121.4 & 2 & 9 & & 4 & 125.0 & 14 & 71 & 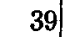 & & \\
\hline 4 & 19 & 135 & 74 & 61 & 1.3 & 10 & 74 & 36 & 38 & 94.7 & 29 & 209 & 110 & & \\
\hline 5 & 40 & 291 & 148 & 143 & 33.5 & 31 & 245 & 122 & 123 & 99.2 & 71 & 536 & 270 & 266 & \\
\hline 6 & 30 & 224 & 120 & 104 & 1 & 22 & 176 & 92 & 84 & 109.5 & 32 & 400 & 212 & 188 & \\
\hline 7 & 30 & 184 & 96 & 88 & 9.1 & 25 & 182 & 86 & ${ }_{2} 96$ & 89.6 & 55 & 366 & 84 & 184 & \\
\hline$\varepsilon$ & 30 & 237 & 115 & 122 & & 16 & 115 & 58 & 57 & 01.8 & 16] & 352 & 73 & & \\
\hline c & 40 & 309 & 16 & 15 & & 7 & 176 & 4 & 32 & 4 & 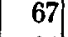 & . & 2 & 39 & \\
\hline & 34 & & & & 85.8 & & 200 & 100 & 100 & 100.0 & & 449 & 215 & 23 & \\
\hline & 38 & 240 & 115 & 125 & 92.0 & 36 & 287 & 138 & 149 & 92. & & 527 & 253 & 27 & \\
\hline 12 & 40 & 314 & 158 & 156 & 101.3 & 31 & 222 & 100 & 12 & 32. & & 536 & 258 & & \\
\hline 12 & 42 & 322 & 127 & 19 & & 32 & 254 & 0 & & 89. & & 576 & 247 & & \\
\hline & 42 & 277 & 125 & 152 & & & 293 & 142 & 151 & 4.1 & & 70 & 267 & 30 & \\
\hline & 40 & 269 & 11 & 150 & & 37 & 313 & & & 04.5 & & & & 30 & \\
\hline & & & & & & 1 & 322 & 148 & 12 & & & & 290 & 37 & \\
\hline & 44 & 328 & 142 & & & 38 & 286 & 135 & & 89.4 & & 614 & 277 & 33 & \\
\hline & 46 & 348 & $1 \overline{0}$ & & & 40 & 320 & 15 & & 90.5 & & 68 & 311 & & \\
\hline & 48 & 40 & & & & 35 & 291 & 12 & & & & & & & \\
\hline & 52 & 353 & 15 & 19 & & 32 & 245 & 124 & 1 & 2.5 & & 698 & 83 & 315 & \\
\hline & $4 \varepsilon$ & 36 & & & & & & & & & & & & & \\
\hline & & & 17 & 20 & & 3 & 350 & 16 & & 87.2 & & & & & \\
\hline & 4 & 376 & 16 & 2 & & 45 & 271 & 1 & & & & & & & \\
\hline & 54 & 35 & 17 & & & 43 & 289 & 148 & & 105.0 & & 85 & & & \\
\hline & 52 & 377 & 169 & 208 & 81.3 & 45 & $|315|$ & 150 & 165 & 90.9 & 97 & 692 & 319 & 373 & 85.5 \\
\hline & & & & & 85.1 & & & & & & & & & & \\
\hline
\end{tabular}

$100 \%$, and that the 106 half-inbred litters had an even higher proportion of males $\left(114.00^{x}: 100 \%\right)$. For the total of 250 litters the sex ratio was 113.2 o' $: 100$ \% .

Until the seventh generation, as already stated, there was no selection of breeding animals in either series. As the sex ratio among the animals in the early generations of the $A$ series was 
TABLE 6

Showing, by generation groups, the sex ratios in the inbred and in the half-inbred letters of the A series (male line)

\begin{tabular}{|c|c|c|c|c|c|c|c|c|c|c|c|c|c|c|c|}
\hline \multirow{2}{*}{ 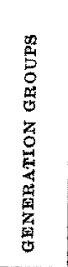 } & \multicolumn{5}{|c|}{$\begin{array}{c}\text { TNBEED } \\
\text { (FIRST AND SECOND XITTERS) }\end{array}$} & \multicolumn{5}{|c|}{$\begin{array}{c}\text { HALFTNBRED } \\
\text { (THIRD AND FOURTH LITTERS) }\end{array}$} & \multicolumn{5}{|c|}{ SUMMARY OF ALL LITTERS } \\
\hline & 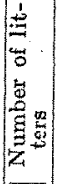 & 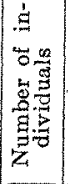 & $\frac{\mathscr{g}}{3}$ & 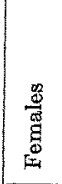 & 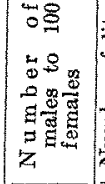 & 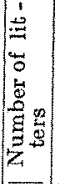 & 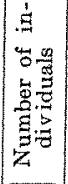 & 遌 & 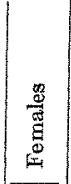 & 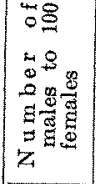 & 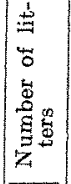 & 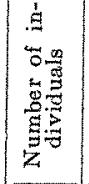 & $\frac{\frac{n_{2}}{3}}{\frac{3}{5}}$ & 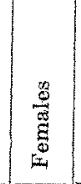 & 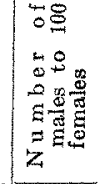 \\
\hline $1-4$ & 46. & 257 & 128 & 129 & 99.2 & $32 \mid$ & 194 & 113 & 81 & & 78 & 451 & & & \\
\hline & 98 & 673 & 360 & 313 & $115^{\circ} .0$ & 74 & 488 & 255 & 233 & & 172 & 1161 & & & \\
\hline $1-7$ & 144 & 930 & 488 & 442 & $\mid 110.4$ & 106 & 682 & 68 & 314 & & 250 & 1612 & 856 & 756 & \\
\hline & 108 & 779 & 412 & 367 & 112.5 & $88 \mid$ & 651 & 336 & 315 & & 196 & 1430 & & $68 ?$ & \\
\hline & & 9 & & & & 11 & 904 & & 13 & & 241 & & & & \\
\hline & & 999 & & 441 & & & 81 & & 69 & & 235 & & & 10 & \\
\hline & & 1144 & 62 & 516 & 121.7 & 119 & 955 & 516 & 439 & & 265 & 2099 & & 55 & \\
\hline & & & 698 & 572 & 122 & 9 & 1020 & & 36 & & 291 & 2290 & & 1058 & \\
\hline & & 1129 & 624 & 505 & 123.5 & 122 & 890 & 482 & 408 & 118.1 & 274 & $\mid 2019$ & 1106 & 913 & | 121.1 \\
\hline & & & 3460 & 2814 & $\begin{array}{r}122.3 \\
\pm 1.55\end{array}$ & & 5230 & & & & 1502 & & $|6260|$ & 5244 & \\
\hline
\end{tabular}

some eight points above the norm, it might appear that inbreeding had tended to increase the relative number of males. Such an interpretation of the results is not warranted, however, since the sex ratio in the litters produced by the mating of unrelated parents was higher than that in the litters obtained by the mating of brother and sister, and since a similar increase in the sex ratio was not found in corresponding litters of the $B$ series (table 7). .

As the females of the seventh generations that were used for breeding were all taken from litters that contained an excess of males, it is among their offspring that we may look for a possible alteration of the sex ratio as a result of selection. The sex ratio in the inbred litters of the eighth generation of the $A$ series was $118.2 \sigma^{7}: 100$ \%. This sex ratio is very much lower than that found in the inbred litters of the seventh generation (150 $0^{\text {* }}$ : 100 \%), but it is still 13 points above the norm (105 $100 \%)$. As examination of the records given in table 4 shows that in 
only one generation (the tenth) after the eighth did the sex ratio for the inbred litters fall to norm, in all other generations it was considerably above the norm, the highest ratio (145.3 $\sigma^{x}: 100$ \%) being found in the litters of the eleventh generation.

While the sex ratios for the inbred litters of the eighth to the twenty-fifth generations varied considerably, the variation was much less after the twelfth generation than before (table 4). A part of this variation was doubtless phenotypic, since seasonal changes in temperature seem to alter the sex ratio in the rat (King and Stotsenburg, '15), and probably also other agencies, such as the age of the mother (King, '16 a), have a similar effect. As all of the sex ratios were relatively high, however, the deviations from the norm cannot be ascribed either to environment or to chance, so they must have been due, in part at least, to the manner in which the breeding animals were selected.

A most striking uniformity in the sex ratios of the inbred litters belonging in the eighth to the twenty-fifth generations of this series is shown by the grouping of the data as made in table 6 . The lowest sex ratio (112.5 o : $100 \%)$ was found in the first group (eighth to tenth generations); the highest sex ratio (130.7 of: 100 \%) appeared in the second group (eleventh to thirteenth generations). Between these extremes there was a difference of only 18 points, while in the four following groups of litters the range of variation in the sex ratios was less than 5 points. For the total of 824 inbred litters the sex ratio was $122.30^{x}: 100$ o . This latter ratio was not due to an abnormal preponderance of males in a few sets of records, but was based on a series of data that in seventeen out of eighteen cases showed an excess of males greater than that considered as normal for the species. The results obtained, therefore, seem to indicate that by selecting breeding animals from litters that contain an excess of males, the sex ratio can be swung in the direction of the selection, although the line is continually inbred, brother and sister. There was in this case, however, no cumulative effect of the selection. The sex ratios were more uniform in the later generations than in the earlier ones, but they were no higher. It is rather an odd coincidence that the sex ratios in the inbred litters of the eighth and of the twenty-fourth generations were exactly the same $\left(118.2 \sigma^{7}: 100\right.$ \% $)$. 
Data given in table 4 show that in the half-inbred litters produced in the eighth to the twenty-fifth generations of the A series the range of variation in the sex ratios was from 99 to 140.8 males for each 100 females, six of these ratios being slightly below the norm. When the data were combined in generation groups (table 6), it was found that not a single group gave a sex ratio as low as the norm. The sex ratios for the litters in the later generation groups were somewhat more uniform than those for the litters in the earlier generation groups, but the uniformity was not as striking as that in the corresponding groups of inbred litters. For the total of 678 half-inbred litters the sex ratio was $115.6 o^{7}: 100$ \% . This ratio was some 11 points above the norm and less than 7 points lower than the sex ratio in the inbred litters belonging to the same group of generations (122.3 $\left.\sigma^{7}: 100 \%\right)$. While the litters produced by the mating of inbred females with outbred stock males thus tended to have a lower sex ratio than did the strictly inbred litters, they did not give the sex ratio that was to be expected according to the current view that chance alone determines whether a male-producing or a female-producting spermatozoön shall fertilize the egg. Such an hypothesis requires that the sexes shall appear in approximately equal numbers when large series of sex:data are examined. In the present case the proportion of the sexes among the 5230 individuals obtained was very far from equal. In only one group (ninth generation) out of eighteen was there a nearly equal proportion of the sexes, in all other groups there was a pronounced excess of males.

The first twenty-five generations of the A series of inbreds comprised 1752 litters containing 13,116 individuals, 7116 males and 6000 females. The sex ratio for this series of animals was 117.4 $\sigma^{r}: 100$ o. This ratio was over 12 points above the norm, and since it was based on data for such a large group of animals, it would seem to indicate that in the rat the sex ratio can be altered by selection within a closely inbred line. In this instance the relative number of males was apparently increased by selecting breeding females from litters that contained an excess of males.

The sex data for the inbred and for the half-inbred litters of the $B$ series, combined in generation groups, are shown in table 7 . 
TABLE 7

Showing, by generation groups, the sex ratios in the inbred and in the half-inbred litters of the $B$ series (female line)

\begin{tabular}{|c|c|c|c|c|c|c|c|c|c|c|c|c|c|c|c|}
\hline \multirow{2}{*}{ 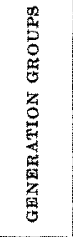 } & \multicolumn{5}{|c|}{$\begin{array}{c}\text { INBRED } \\
\text { (FIRST AND SECOND LIPTERS) }\end{array}$} & \multicolumn{5}{|c|}{$\begin{array}{c}\text { HALF-TNBRED } \\
\text { (THIRD AND FOURTH LITTERS) }\end{array}$} & \multicolumn{5}{|c|}{ SUMMARY OF ALI LITTERS } \\
\hline & 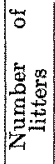 & 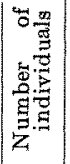 & 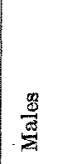 & 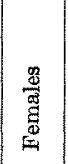 & 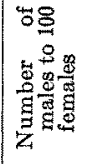 & 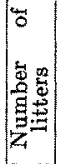 & 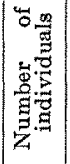 & 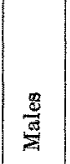 & 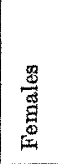 & 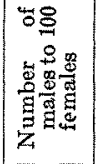 & 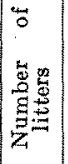 & 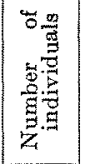 & 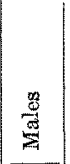 & 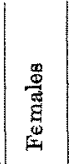 & 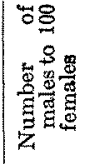 \\
\hline $1-4$ & 36 & 233 & 122 & 111 & & 15 & 109 & 53 & 56 & & 51 & 42 & & & \\
\hline $5-7$ & 100 & 699 & 364 & 335 & 108.7 & 78 & 603 & 300 & 303 & 99.0 & 178 & 1302 & 664 & 638 & 104 \\
\hline $1-7$ & 136 & 932 & 486 & 446 & 109.0 & 93 & 712 & 353 & 359 & 98.3 & 229 & 1644 & 839 & 805 & 104 \\
\hline & & 795 & & & & 73 & 91 & 52 & 239 & 105.4 & 177 & & & 52 & \\
\hline & 120 & 876 & 400 & 476 & 84.0 & 99 & 763 & 358 & 405 & 88.4 & 219 & 1639 & 758 & 881 & 86 \\
\hline & 130 & 888 & 386 & 502 & 76.9 & 116 & 928 & 450 & 478 & 94.1 & 246 & 1816 & 836 & 980 & 85 \\
\hline & 138 & 1083 & 472 & 611 & 77.3 & 113 & 897 & 414 & 483 & 85.7 & 251 & 1980 & 886 & 1094 & 80 \\
\hline & 152 & 1102 & 505 & 597 & 84.6 & 109 & 845 & 401 & 444 & 90.3 & 261 & 1947 & 906 & 1041 & 87 \\
\hline $23-25$ & 150 & 1149 & 506 & 643 & 78.7 & 123 & 875 & 414 & 461 & 89.8 & 273 & 2024 & 920 & 1104 & 83 \\
\hline & 794 & 5893 & 2651 & 3242 & $\begin{array}{r}81.8 \\
+1.56\end{array}$ & 633 & 4799 & 2289 & 2510 & $\begin{array}{r}91.1 \\
\pm 1.74\end{array}$ & 1427 & 10692 & 4940 & 5752 & $\begin{array}{r}85 . \\
+1.3\end{array}$ \\
\hline
\end{tabular}

In the $B$ series, as in the $A$ series, there was a wide range of variation in the sex ratios of the litters produced in the first seven generations (table 5). When the data were combined in generation groups (table 7), the sex ratio in the 136 inbred litters (109 $o^{7}: 100$ ) was found to be above the norm, while that in the half-inbred litters (98.3 $\sigma^{x}: 100$ \%) was below the norm. These two ratios so nearly balance each other that for the total of 229 litters the sex ratio was $104.2 \sigma^{7}: 100 \%$, or less than 1 point below the norm: in the corresponding litters of the $A$ series the sex ratio was 8 points above the norm (113.2 of: 100 o). On combining the records for the first seven generations of the two inbred series (A, B), it was found that the total of 479 litters gave a sex ratio of 108.6 o : 100 . While this ratio is over 3 points above the norm, it is not sufficiently high to warrant the conclusion that the normal sex ratio was changed through inbreeding, particularly as the ratio was due in great part to an unusual excess of males in the hal-inbred litters of the A series (table 4 ). 
As far as can be judged from the results of this part of the investigation, close inbreeding, even when the animals are poorly nourished, does not increase the proportion of male offspring to any extent.

The breeding females in the seventh generation of the $\mathrm{B}$ series of inbred were all taken from litters that contained an excess of females; among their offspring the sex ratio was $94.3 \sigma^{7}: 100$ ㅇ․ In not one of the subsequent generations was the sex ratio in the inbred litters as high as the norm, the nearest approach to the norm was in the twelfth generation, where the sex ratio was $101.3 \sigma^{7}$ : 100 \& (table 5). 'In these inbred litters, as in the corresponding ones of the A series, the sex ratios were more uniform in the later than in the earlier generations, but there was no cumulative effect of selection in either case. In the $B$ series, after the thirteenth generation, there was very little change in the relative proportion of the sexes from one generation to the next, and some of the variation found, as stated for the A series, can doubtless be ascribed to environmental action.

When the data for the inbred litters of the eighth to the twentyfifth generations of the B series were combined in generation groups (table 7), it was found that the sex ratios for the various groups showed even greater deviations from the norm than did those for corresponding litter groups in the A series, but that this deviation was in the reverse direction, i.e., the number of females born greatly exceeded the number of males. The highest sex ratio for any group in the B series was $92.5 o^{7}: 100$ o : for the entire group of 794 litters the sex ratio was $81.8 o^{\pi}: 100$ o , or 23 points below the norm. This latter ratio is far too low to be considered as a chance variation, and it certainly cannot be attributed to the action of environment. For both series of inbreds were reared simultaneously under the same environmental conditions, and if one ventured to suggest that environment swung the sex ratio in the $B$ series towards the female side it would be necessary to assume that the same environment acted on the animals of the A series in a reverse direction and so swung the sex ratio towards the male side. 
As the sex ratio for the inbred litters of the B series was 23 points below the norm, while that for corresponding litters of the A series was 18 points above the norm, it would appear that the sex ratio in the rat can be swung by selection farther towards the female side than towards the male side. Moenkhaus ('11) obtained a similar result in his inbreeding experiments with Drosophila.

The half-inbred litters in the eighth generation of the B series gave a sex ratio nearly 10 points higher than the norm, so here selection was not effective at once in changing the sex ratio. In none of the subsequent generations, however, was the sex ratio in these litters above the norm, most of them were considerably below it (table 5). When the data were combined in generation groups (table 7), it was found that the sex ratios for all groups except one (eighth to tenth generations) were very low. For the total of 633 litters the sex ratio was $91.1 o^{7}: 100$ o thus being 14 points below the norm and 9 points higher than the sex ratio for the inbred litters of this series.

In each of the inbred series the sex ratiosin the half-inbred litters belonging in the eighth to the twenty-fifth generations showed less deviation from the norm than did the sex ratios in the corresponding inbred litters, yet in each case the difference between the sex ratio for the inbred group of litters and that for the halfinbred group was less than the difference between the sex ratio for the half-inbred litters and the norm. The possible significance of these results will be discussed later.

In order to obtain the sex ratios for the various generations of the inbred strain as a whole, the data for the two series (A, B) were combined as shown in table 8 .

The range of variation in the sex ratios of the litters in the first four generations of the inbred strain was greater than that among all of the other generation groups (table 8). This result was to be expected, considering the relatively small number of individuals in these generations and the adverse conditions under which the animals lived. When the data were combined, however, the sex ratio obtained (110.3 o : 100 o) was only 5 points above the 
TABLE 8

Showing the sex data for each of the first twenty-five generations of the inbred strain (series $A, B$ ), also the sex ratios when the data were combined in

\begin{tabular}{|c|c|c|c|c|c|c|}
\hline $\begin{array}{l}\text { GENERA- } \\
\text { TIONS }\end{array}$ & $\begin{array}{l}\text { NOMBER OF } \\
\text { LITTERS }\end{array}$ & $\begin{array}{l}\text { NUMEER OF } \\
\text { INDIVIDUALS }\end{array}$ & MaLES & TEMALES & $\begin{array}{l}\text { NUMBER OF } \\
\text { MALES TO } 100 \\
\text { FEMALES }\end{array}$ & $\begin{array}{c}\text { NOMBER OF } \\
\text { MALES TO } 100 \\
\text { FEMALES } \\
\text { IN GENERATION } \\
\text { GROUPS }\end{array}$ \\
\hline 1 & 5 & 34 & 21 & 13 & 161.5 & \\
\hline 2 & 20 & 137 & 65 & 72 & 90.3 & \\
\hline 3 & 36 & 180 & 90 & 90 & 100.0 & \\
\hline 4 & 68 & 442 & 240 & 202 & $118: 8$ & 110.3 \\
\hline 5 & 132 & 952 & 470 & 482 & 97.5 & \\
\hline 6 & 107 & 745 & 392 & 353 & 111.0 & \\
\hline 7 & 111 & 766 & 417 & 349 & 119.5 & 108.0 \\
\hline 8 & 103 & 772 & 402 & 370 & 108.6 & \\
\hline 9 & 129 & 910 & 466 & 444 & 104.9 & \\
\hline 10 & 141 & 1034 & 514 & 520 & 98.8 & 103.6 \\
\hline 11 & 154 & 1140 & 594 & 546 & 108.8 & \\
\hline 12 & 147 & 1127 & 586 & 541 & 108.3 & \\
\hline 13 & 159 & 1229 & 609 & 620 & 98.2 & 104.8 \\
\hline 14 & 161 & 1215 & 619 & 596 & 103.9 & \\
\hline 15 & 161 & 1255 & 649 & 606 & 107.1 & \\
\hline 16 & 159 & 1155 & 567 & 588 & 96.4 & 102.5 \\
\hline 17 & 184 & 1459 & 728 & 731 & 99.6 & \\
\hline 18 & 166 & 1307 & 660 & 647 & 102.0 & \\
\hline 19 & 166 & 1313 & 642 & 671 & 95.7 & 99.1 \\
\hline 20 & 178 & 1294 & 671 & 623 & 107.7 & \\
\hline 21 & 184 & 1441 & 717 & 724 & 99.0 & \\
\hline 22 & 190 & 1502 & 750 & 752 & 99.7 & 101.8 \\
\hline 23 & 170 & 1319 & 663 & 656 & 101.1 & \\
\hline 24 & 190 & 1886 & 689 & 697 & 98.9 & \\
\hline \multirow[t]{2}{*}{25} & 187 & 1338 & 674 & 664 & 101.5 & 100.4 \\
\hline & 3408 & 25452 & 12895 & 12557 & $\begin{array}{l}102.7 \\
\pm 1.28\end{array}$ & \\
\hline
\end{tabular}


norm. The sex ratios in the litters of the fifth to the twentyfifth generations varied from 95.7 to 119.5 males to each 100 females. Variation, it will be noted, was around the norm, eight of the twenty-one ratios being at or above the norm, the rest below it. When combined in generation groups the sex data gave a very uniform series of ratios, as the last column of table 8 shows -not one of these ratios varied more than 6 points from the norm. A variation as great as this would doubtless be found in the sex ratios of any other large series of albino rats, regardless of the manner in which the animals were bred. For the 3256 individuals comprised in the first seven generations of the inbred strain the sex ratio was $108.6 \sigma^{7}: 100 \%$. This ratio is sufficiently close to the norm, I think, to indicate that, in the rat, inbreeding per se does not produce a marked increase in the number of male offspring. The sex ratio in the 22,196 individuals in the remaining eighteen generations was $101 o^{7}: 100$ of : for the entire series of 25,452 animals in the inbred strain the sex ratio was $102.7 o^{7}: 100$ o. While these last two ratios are slightly below the norm, it is evident that in the inbred strain as a whole the sex ratio was not greatly influenced either by inbreeding or by selection. The very different sex ratios obtained in the two series of the inbred strain seem to show, however, that through selection the one inbred strain was separated into two distinct lines, one line (A) having a tendency to produce an excess of males, the other line (B) tending to produce a preponderance of females.

Unfortunately, one cannot prediet with certainty what the sex ratio will be in the litters cast by any given inbred female, neither does the sex ratio in the litters cast by one female give a clear indication regarding the proportion of the sexes that will be found among the offspring of a sister rat. It is only by taking the averages for a large number of litters in a given series that the change in the sex ratio is made manifest. As an illustration of the individual differences in females regarding their tendencies to cast young of a certain sex, four sets of data for litters cast by sister females are shown in table 9 . In each case given, sister rats were first paired with the same litter brother and later with the same stock male. 
TABLE 9

Showing the difference between inbred sisters regarding their tendency to produce an excess of male or of female young when mated with the same male

\begin{tabular}{|c|c|c|c|c|c|c|c|c|c|}
\hline $\begin{array}{l}\text { LITTER } \\
\text { SERIES }\end{array}$ & $\begin{array}{c}\text { NUMBER } \\
\text { OF } \\
\text { YOVNG }\end{array}$ & MALES & FEMALES & SIRE & $\begin{array}{l}\text { LITTER } \\
\text { SERTES }\end{array}$ & $\begin{array}{c}\text { NUMBER } \\
\text { OF } \\
\text { xOUNG }\end{array}$ & MaLes & FEMALES & StRE \\
\hline \multicolumn{10}{|c|}{1} \\
\hline \multicolumn{5}{|c|}{$11 B_{73}$} & \multicolumn{5}{|c|}{$11 B_{74}$} \\
\hline 1 & 11 & 3 & 8 & $11 B_{78}$ & 1 & 10 & 6 & 4 & $11 B_{78}$ \\
\hline 2 & 11 & 5 & 6 & $11 B_{78}$ & 2 & 11 & 8 & 3 & $11 B_{78}$ \\
\hline \multirow[t]{3}{*}{3} & 10 & 2 & 8 & Stock & 3 & 11 & 7 & 4 & Stock \\
\hline & & & & & 4 & 9 & 5 & 4 & Stock \\
\hline & 32 & 10 & 22 & & & 41 & 26 & 16 & \\
\hline \multicolumn{10}{|c|}{2} \\
\hline \multicolumn{5}{|c|}{$17 B_{14}$} & \multicolumn{5}{|c|}{$17 \mathrm{~B}_{15}$} \\
\hline 1 & 8 & 3 & 5 & $17 \mathrm{~B}_{16}$ & 1 & 10 & 4 & 6 & $17 B_{16}$ \\
\hline 2 & 9 & 4 & 5 & $17 \mathrm{~B}_{16}$ & 2 & 10 & 3 & 7 & $17 \mathrm{~B}_{16}$ \\
\hline 3 & 8 & 3 & 5 & Stock & 3 & 7 & 3 & 4 & Stock \\
\hline \multirow[t]{2}{*}{4} & 3 & 1 & 2 & Stock & 4 & 11 & 5 & 6 & Stock \\
\hline & 28 & 11 & 17 & & & 38 & 15 & 23 & \\
\hline \multicolumn{10}{|c|}{3} \\
\hline \multicolumn{5}{|c|}{$12 A_{194}$} & \multicolumn{5}{|c|}{$13 A_{155}$} \\
\hline 1 & 8 & 6 & 2 & $12 \mathrm{~A}_{136}$ & 1 & 8 & 3 & 5 & $12 \mathrm{~A}_{136}$ \\
\hline 2 & 8 & 4 & 4 & $12 A_{136}$ & 2 & 9 & 4 & 5 & $12 \mathrm{~A}_{136}$ \\
\hline 3 & 7 & 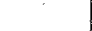 & 7 & Stock & 3 & 5 & 4 & 1 & Stock \\
\hline \multirow[t]{2}{*}{4} & 9 & 3 & 6 & Stock & 4 & 9 & 5 & 4 & Stock \\
\hline & 32 & 13 & 19 & & & 31 & 16 & 15 & \\
\hline \multicolumn{10}{|c|}{4} \\
\hline \multicolumn{5}{|c|}{$13 \mathrm{~A}^{2}{ }_{4 \mathrm{E}}$} & \multicolumn{5}{|c|}{$13 A_{46}^{2}$} \\
\hline 1 & 9 & 5 & 4 & $13 \mathrm{~A}_{48}^{2}$ & 1 & 7 & 5 & 2 & $13 A_{48}^{2}$ \\
\hline 2 & 10 & 5 & 5 & $13 \mathrm{~A}_{48}^{2}$ & 2 & 9 & & 9 & $13 \mathrm{~A}_{43}^{2}$ \\
\hline 3 & 10 & 5 & 5 & Stock & 3 & 12 & 7 & 5 & Stock \\
\hline 4 & 10 & 5 & 5 & Stock & 4 & 8 & 5 & 3 & Stock \\
\hline & 39 & 20 & 19 & & & 36 & 17 & 19 & \\
\hline
\end{tabular}


The first set of records given in table 9 shows the very great difference in the sex tendencies of two sister rats belonging in the $B$ series. Female $11 B_{73}$ had cast three litters when she developed pneumonia and had to be killed. Each of these litters contained such a large excess of females that among her thirty-two offspring the sex ratio was only $45.4 \sigma^{7}: 100 \%$ : Female $11 B_{74}$, on the other hand, showed a very strong tendency to produce male young, whether she was paired with a brother or with a stock male; among her forty-one offspring the sex ratio was $162.5 \sigma^{\pi}: 100$ ㅇ. As yet no other sister rats have shown such a pronounced difference in their sex tendencies.

A very great similarity in the sex tendencies of sister rats is shown by the second set of records in table 9 . Each litter cast by $17 \mathrm{~B}_{14}$ and by $17 \mathrm{~B}_{15}$ contained an excess of female young, whether the sire of the litter was an inbred or a stock male. In each group of litters the sex ratio was about 65 o $^{7}: 100$ o .

Female $12 \mathrm{~A}_{13^{4}}$ produced an excess of male young in each of the two litters sired by her brother, but the two litters sired by a stock male showed a very great excess of female young. Conversely, while female $12 \mathrm{~A}_{135}$ cast more female than male young when paired with a brother, she showed a strong tendency to produce an excess of male young when mated with a stock male.

The last set of records in table 9 shows a case where the total number of offspring produced by each of two sister rats contained a nearly equal proportion of the sexes, but this proportion was attained in very different ways. Female $13 \mathrm{~A}_{45}^{2}$ showed a most pronounced tendency to produce an equal number of male and female young in each of her four litters. In the litters of female $13 \mathrm{~A}_{46}^{2}$ the sexes were very unequally distributed; one litter of nine young consisted entirely of females - a most unusual phenomenon in a litter of such size.

Numerous other cases, similar to the ones given, could be furnished from the records for these inbred rats. The eases cited are sufficient, I think, to show the individual differences in the females regarding their tendencies to cast male or female offspring. Incidentally, these records show, also, that the female plays a more important rôle in determining the sex ratio than is generally believed. 
An examination of the sex data for successive litters cast by many hundreds of female rats does not indicate that there is "a change of sex tendency from litter to litter" in the female, as Papanicolau ('15) states is the case in guinea-pigs. Such a tendency is not shown in any of the cases given in table 8 , and while the sex-proportions in the litters do change in many cases, the change is not general or striking enough to warrant the conclusion that there is a definite sex-determining factor involved.

4. THE SEX RATIOS IN THE LITTERS OBTAINED BY THE MATING OF STOCK FEMALES WITH INBRED MALES

As a check for the results obtained by the mating of inbred females with stock males, series of stock females were bred to males from various generations of the inbred strain. The number of such experiments was small, considering the scale on which the main series of experiments was conducted, but the results obtained were uniform enough to be significant.

The stock females used in these experiments were reared under the same environmental conditions as the inbred rats. When they were about three months old they were paired with males from the $\mathrm{A}$ or from the $\mathrm{B}$ series that had sired inbred litters. In order to make this series of records more strictly comparable to that obtained in the inbred strain, only four litters from any one female were recorded.

The data for the litters obtained by the mating of stock females with inbred males are given in table 10.

Stock females paired with males from the fifth generation of the inbred strain produced litters in which the sex ratio was below the norm, whether the sire of the litter belonged to the A or to the B series of inbreds. The litters sired by males from A series, however, had a much higher sex ratio than did those sired by males from the $B$ series, although at the fifth generation there was no selection of breeding animals in the inbred strain according to a definite plan. The eighteen litters in this series gave a sex ratio of $94.7 \sigma^{7}: 100$ o , or 10 points below the norm. This ratio might seem to indicate that inbred males tended to produce an excess of female offspring, but the number of litters 
TABLE 10

Showing the sex ratios in litters produced by the mating of outbred stock females with inbred males

\begin{tabular}{|c|c|c|c|c|c|c|}
\hline $\begin{array}{c}\text { XNBRED } \\
\text { SERIES TO } \\
\text { WHICH SIRES } \\
\text { BEEONIEDD }\end{array}$ & $\begin{array}{c}\text { GENERAATION } \\
\text { TO WHIOH } \\
\text { SIRES } \\
\text { BELONGED }\end{array}$ & $\begin{array}{l}\text { NUMBER of } \\
\text { LITTERS }\end{array}$ & $\begin{array}{c}\text { NUMBER OF } \\
\text { INDTVIDUALS }\end{array}$ & MALIES & FEMALES & $\begin{array}{l}\text { NUMBBRR OF MALES } \\
\text { TO } 100 \text { FEMALES }\end{array}$ \\
\hline A & 5 & 10 & 59 & 30 & 29 & 103.5 \\
\hline \multirow[t]{2}{*}{ B } & 5 & 8 & 52 & 24 & 28 & 85.7 \\
\hline & & 18 & 111 & 54 & 57 & $94.7 \pm 4.30$ \\
\hline A & 9 & 7 & 51 & 28 & 23 & 121.7 \\
\hline A & 12 & 12 & 60 & 30 & 30 & 100.0 \\
\hline A & 13 & 5 & 33 & 20 & 13 & 153.9 \\
\hline A & 15 & 19 & 177 & 79 & 98 & 80.6 \\
\hline A & 16 & 11 & 126 & 67 & 59 & 113.6 \\
\hline A & 17 & 14 & 117 & 60 & 57 & 105.2 \\
\hline \multirow[t]{2}{*}{ A } & 18 & 39 & 347 & 177 & 170 & 104.1 \\
\hline & & 107 & 911 & 461 & 450 & $102.3 \pm 5.88$ \\
\hline B & 10 & 12 & 97 & 51 & 46 & 110.9 \\
\hline B & 12 & 11 & 75 & 38 & 37 & 102.7 \\
\hline B & 13 & 8 & 42 & 18 & 24 & 75.0 \\
\hline B & 15 & 19 & 172 & 87 & 85 & 102.3 \\
\hline B & 16 & 29 & 243 & 116 & 127 & 91.3 \\
\hline \multirow[t]{2}{*}{ B } & 18 & 38 & 313 & 152 & 161 & 94.4 \\
\hline & & 117 & 942 & 462 & 480 & $96.2 \pm 30.14$ \\
\hline Total. . & & 242 & 1964 & 977 & 987 & 99.1 \\
\hline
\end{tabular}

examined was too small to warrant any general conclusion from the results obtained.

The second section of table 10 shows the sex ratios in the various groups of litters obtained from the matings of stock females with males from the ninth to the eighteenth generations of the A series of inbreds. There was considerable variation in these sex ratios, as was to be expected considering the number of animals involved. The total of 107 litters in this group gave a sex ratio of $102.3 \sigma^{7}: 100$ \%. This ratio, it will be noted, was below the norm, although the sires of the litters were males that, paired with their sister, had fathered litters in which there was, as a rule, a preponderance of male young. 
The sex ratios in the various groups of litters obtained by the mating of stock females with males from the tenth to the eighteenth generations of the $B$ series of inbreds showed a much narrower range of variation than that found in the litters sired by males of the A series of inbreds, although the number of litters produced in the two series was about the same. The 117 litters in this group gave a sex ratio of $96.2 o^{7}: 100$ o , which was 9 points below the norm. Any significance that this ratio might seem to have, when taken alone, is apparently annulled by the fact that the sex ratios for the other litters groups were also below the norm, whether the sires of the litters came from the $\mathrm{A}$ or from the $\mathrm{B}$ series of inbreds. Moreover, the probable error of the mean, calculated from the averages for the various sets of litters, was so large in every case that the differences between the sex ratios of the various groups were rendered valueless.

The 242 litters in this series gave a sex ratio of $99.1 o^{7}: 100$ o While this ratio was some 6 points below the norm, it differed by only 4.4 points from the sex ratio found in the 1510 litters obtained by the mating of inbred females with stock males $\left(103.5 \sigma^{7}\right.$ $: 100$ o ). The results as a whole, therefore, do not indicate that the sex ratio was influenced to any extent by the fact that the sires of the litters were inbred rather than outbred males.

The final experiment to be made, the pairing of females from the one inbred series with males from the other inbred series, was not begun until the animals reached the twenty-sixth generation. The number of litters as yet obtained is too small to afford a basis for any general conclusion, but thus far females of the $A$ series (male line) when paired with males from the B series (female line) have produced more male than female young, and, conversely, females of the $B$ series, when paired with males of the $A$ series, have shown a tendency to cast more female than male young.

The results of these various series of experiments are summarized and discussed in the following section. 


\section{DISCUSSION}

As a basis for discussion the results obtained in this investigation are briefly summarized as follows:

1. The inbreeding of litter brother and sister for six consecutive generations, during which there was no selection of animals for breeding, did not increase the number of male offspring to any extent. The sex ratio in the 3256 young obtained was $108.6 \mathrm{o}^{x}$ : $100 \%$, or less than 4 points above the sex ratio taken as the norm (105 o : 100 o ).

2. Beginning with the seventh generation all breeding females in the A series were taken from litters that contained an excess of males. After this time the females in this series tended to produce an excess of male young, whether they were paired with a litter brother or with an unrelated stock male (table 6). The litters sired by inbred males gave a sex ratio of $122.30^{7}: 100$ 우, or over 17 points above the norm; while the litters sired by stock males showed a sex ratio of 115.6 or $: 100$ o , or nearly 11 points above the norm.

3. From the time that the breeding females in the $B$ series were selected from litters containing an excess of females (seventh generation), the litters produced showed a reverse proportion of the sexes to that shown by corresponding litters in the A series (table 7). Litters sired by inbred males had a very low sex ratio $(81.8$ a : 100 o); in the litters sired by stock males the sex ratio was 9 points higher than that in the inbred litters (91.1 $\sigma^{7}: 100$ o ), but it was still significantly lower than the norm.

4. On combining the data for the two inbred series it was found that among the 25,452 individuals comprised in the inbred strain the sex ratio was $102.7 \sigma^{7}: 100 \%$, or less than 3 points below the norm (105.0 of : 100 \&). It thus appears that through selection the inbred strain was separated into two distinct lines: one (A) showing a high sex ratio, the other $(B)$ a low sex ratio. Selection had the greater influence on the female line, since the sex ratio for the litters of the B series showed greater deviation from the norm than did that for the litters of the $A$ series.

5. Stogk females mated with inbred males tended to produce litters in which the sex ratio was below the norm, regardless of 
whether the male belonged to the $\mathrm{A}$ or to the $\mathrm{B}$ series of inbreds. The litters sired by males from the A series showed a higher sex ratio (102.3 on : 100 o ), however, than did the litters sired by males from the B series (96.2 $\left.\sigma^{7}: 100 \%\right)$, but these ratios are not significant, since they differ from each other and from the norm by less than three times the probable error (table 10).

Düsing's contention that close inbreeding increases the relative number of male offspring was based mainly on statisties of human births collected from several isolated communities in which there were many consanguineous marriages, and on the supposedly great preponderance of male births among the Jews, who are a clannish race and intermarry more frequently than do other civilized races. The latter evidence is undoubtedly invalid, as Pearl and Salaman ('13) have shown that the normal sex ratio among the Jews is the same as that in other races of man (105 o : 100 o ), and that the anomalous sex ratio among them is due to faulty registration, male births being recorded where those of females are not. The high sex ratio in the other cases cited by Düsing can doubtless be attributed to a similar cause. The great excess of males found in various strains of thoroughbred dogs Heape ('08) ascribed in part to inbreeding, but in these cases also it is probable that the statistics are not reliable, since female pups are commonly discarded from large litters and males are registered more often, as a rule, than females.

The inbreeding experiments of Huth ('87) on the rabbit, of Schultze ('03) and of Copeman and Parsons ('04) on mice were made with relatively small numbers of animals, and the sex ratios obtained showed no greater deviations from the norm than might have been expected under the conditions of the experiments. Shull ('13) found no change in the sex ratio of Hydatina senta as a result of repeated inbreeding, the proportion of male-producers and of female-producers remaining practically constant. In the present series of inbreeding experiments with the albino rat, all of the animals belonging to the earlier generations suffered severely from malnutrition, which Düsing ('84) considered as a very potent factor in increasing the number of male offspring, yet among the individuals in the first seven generations the sex ratio was only 
slightly higher than the norm. The results of these various series of experiments would seem to indicate that inbreeding per se has little, if any, effegt on the sex ratio.

Moenkhaus' ('11) extensive series of inbreeding experiments on Drosophila so elosely parallel my own experiments on the rat, both in the manner in which the experiments were conducted and in the results obtained, that a brief résumé of his work must be given here.

In order to obtain the normal sex ratio in Drosophila, Moenkhaus ascertained the sex of 26,933 imagos that developed from eggs laid by wild flies, and found among them a sex ratio of 88.8 $o^{7}: 100$ o. In this species, therefore, there is normally an excess of females, as other investigators (Rawls, '13; Hyde, '14; Warren, '18) have noted. The experiments were conducted in the following way: "Two pairs were selected from nature, the one showing a high, the other a low female ratio. These were selected as the parents of the two strains to be developed. From among the offspring of each of these two pairs a number of single matings were made. From among these the pair that showed the most favorable ratio in the desired direction was selected to continue the strain. The same process was repeated as often as desired."

In this way Moenkhaus developed two inbred strains in one of which the individuals showed a high sex ratio, in the other a low sex ratio. The results of this part of the investigation showed that "it is possible to develop a strain with a high female ratio much more easily and pronouncedly than a male strain." Moenkhaus then made reciprocal crosses between the two strains in order to determine, "first, whether the maternal or the paternal elements had an equal share in the control of this ratio, and second, whether this ratio was determined in the process of fertilization." The experiments showed, in a most decided way, that "the male has little or no influence in determining the sex ratio in this species. In most of the cases the ratio of the offspring falls pretty elosely around that of the strain from which the females were taken. . . . It is not certain, however, that the sex ratio is established before fertilization, since the experiments do not with certainty 
entirely exclude the male influence." In his summary Moenkhaus states: "The sex ratio is one of the qualities that is, like color, an inherent character of this creature, strongly transmissible and amenable to the process of selection. . . . Sex is probably very little, if at all, influenced at fertilization in this species, but it is probably determined much earlier and by the female." Moenkhaus' conclusions regarding the character of the sex ratio and its amenability to selection are as applicable to the rat as they are to Drosophila, judging from the results of my inbreeding experiments on the former species. Neither of these investigations, however, give any information regarding the causes that condition sex, although each seems to indicate that the female takes quite as important a part in this process as does the male.

In the inbred strain, after the animals for breeding were selected in each generation according to a definite plan, the two series (A and $B$ ) became two separate lines as regards the sex-proportions among the young. In the one line (A) the litters contained, as a rule, an excess of males; in the other line (B) there was a corresponding excess of females. Between these two lines there was no very marked difference as regards the size of the individuals at a given age, their fertility or longevity, as the data given in previous papers have shown (King, '18, '18 a). Generation after. generation, as far as the experiments have been carried, the sex ratios in the inbred lines have remained distinct, and the variations from the norm have been in the same direction in each generation of each series. These results are definite enough, and they are based on data from a sufficiently large number of animals. I think, to warrant the conclusion that in the rat the sex ratio is to a certain extent at least, a character that is amenable to selection.

\footnotetext{
'Warren ('18) has recently repeated Moenkhaus' selection experiments on Drosophila, and concludes that the sex ratio in this form is "not readily, if at all, modifiable by selection." Warren believes that the modified sex ratios found in two of his three series of experiments were due to 'chance variation,' and he attributes the anomalous sex ratios obtained by Moenkhaus to the action of a sex-linked lethal factor - the explanation offered by Morgan ('14 a) to account for the unusually low sex ratios found in several strains of Drosophila.
} 
As the rats had been inbred brother and sister only, they were, according to Fish's ('14) calculations, 79.687 per cent homozygous' at the time that the selection of breeding animals began (seventh generation). Selection, if effective at all in changing the sex ratio, should act in one or two generations, unless a considerable number of factors were involved. In the latter case selection might produce a gradual change in the sex ratio which would reach its culmination only after a number of generations. In each series, as table 4 and table 5 show, the sex ratio in the inbred litters of the eighth generation was close to the sex ratio that was the average for all of the litters produced in the eighth to the twenty-fifth generations, and the sex ratios in the later generations showed no greater deviation from the norm than did those in the earlier generations, although they were somewhat more uniform. Selection thus produced its maximum effect at once, and could not shift the centre of gravity of the variation in the direction of the selection, as it did in the experiments which Castle and Phillips ('14) made with piebald rats. It would appear from these results that very few heritable factors concerned in the production of the sex ratio, possibly not more than a single pair, were acted upon by selection, and that, as Pearl ('17) has stated: "selection acts only as a mechanical sorter of existing diversities in the germ plasm and not as a cause of alteration in it."

As sister rats show such marked individual difference regarding their sex tendencies, and as both nutritive (Slonaker and Card, '18) and environmental conditions (King and Stotsenberg, '15) seem to influence the sex ratio in the rat, it would seem that the sex ratio may be modified by so many agencies that it would be useless to attempt to determine the number or the nature of the particular factors that were acted upon by selection in the present case. The factors involved are evidently not of very great potency, and their action is clearly shown only when a relatively large number of animals are closely inbred under environmental and nutritive conditions that are as uniform as it is possible to make them. Whatever their nature, or in whatever manner they may be inherited, I believe that these factors act on the metabo- 
lism of the ova in such a way as to render the ova more easily fertilized by one kind of spermatozoa than by the other. In the A series of inbreds, under the conditions given, the ova tended to attract spermatozoa that were 'male-producing;' in the B series, the ova tended to attract spermatozoa that were 'female-producing.'

In advocating the possibility that fertilization may be selective I am aware that I run counter to the general belief that any egg is capable of fertilization by any spermatozoön that happens to come in contact with it, and that those whose views have much weight in molding biological opinion believe that this hypothesis is "so improbable as almost to invalidate any interpretation into which it enters" (Wilson, '10). Just why this hypothesis is considered so untenable is not clear. It is true that it has not been definitely proved in any case, but neither has it been disproved, nor has any convineing proof been offered, as yet, for the very elaborate hypotheses that have been advanced to account for heredity in general and in specific cases. The burden of proof rests equally upon those who object to this hypothesis as on those who maintain it.

We owe to MeClung ('02) the suggestion that the accessory chromosome may be a sex-determinant. In discussing the possible action of the accessory chromosome in determining sex, MeClung ('02 a) states: "even up to the time of fertilization the female elements are so placed as to react readily to stimuli from the mother. Here they are approached by the wandering male elements from which they may choose-if we may use such a term for what is probably chemical attraction-either the spermatozoa containing the accessory chromosome or those from which it is absent. In the female element, therefore, as in the female organism, resides the power to select that which is for the best interest of the species."

In advocating selective fertilization as the probable cause of anomalous sex ratios, Heape ('09) says: "it must be remembered that there are an enormous number of spermatozoa available for the fertilization of each ovum, and, moreover, it will be recollected there are undoubtedly chemotactic properties associated 
with ova which insure that ova of different species floating in the sea shall each be fertilized by spermatozoa of the same species, so that to grant there is still more delicate chemotaxis at work is not an illegitimate but is indeed a reasonable supposition." Castle ('03) also postulated selective fertilization in the elaboration of his Mendelian theory of sex-determination.

The one attempt that has been made to test the hypothesis of selective fertilization (Morgan, Payne and Browne, '10) seemed to indicate that the egg is fertilized by the first spermatozoön that strikes it 'head-on,' but the conditions under which the observations were made were so abnormal that no definite conclusions from them were possible, and even Morgan ('11) states that the evidence is 'admittedly insufficient.'

An earlier experiment that has a bearing in this connection seems to have been overlooked and therefore needs to be noted here. Marshall ('10) injected into the vagina of a pure-bredDandieDinmont bitch a mixture of seminal fluid taken from a pure-bred dog of the same species and from a mongrel terrier of unknown ancestry. Fifty-nine days later the bitch littered, producing four pups which were much alike. One of the pups died early, but as the other three developed into mongrels which resembled the terrier sire there was little doubt but that all four puppies were mongrels. Marshall cites another case in which a Dandie Dinmont bitch copulated with a dog of the same breed and two days later with a Scotch terrier. The bitch littered three pups; one was pure Dandie Dinmont, the other two half-bred Scotch terriers. These cases, according to Marshall, were indicative of a 'selective' on the part of the ova of the pure-bred bitch to "conjugate with dissimilar rather than with related spermatozoa."

I have recently been making a series of experiments somewhat on the order of those cited by Marshall, and the results obtained indicate a very strong tendency on the part of the ova of the albino rat to conjugate with spermatozoa from the wild gray rat rather than with the spermatozoa of the albino rat, although under the conditions of the experiment, details of which will be published later, the advantage in every case was with the spermatozoa from the albino male. If fertilization can be selective in such 
cases I can see no valid objection to the assumption that the chemotactic reaction between ova and spermatozoa may be even more delicate and thus, under given conditions, make possible the fertilization of an egg by a spermatozoön that has one sex potency rather than the other.

There is another possible interpretation of the anomalous sex ratios found in the inbred litters of the two series. We might assume that inbreeding had acted on the males in some way so as to render one kind of spermatozoön more potent than the other in fertilizing the ova, and that this difference in potency came to have an heritable basis in the germ plasm and so could be acted upon by selection. In the $A$ series of inbreds, according to this assumption, the 'male-producing' spermatozoa became the more potent; in the B series the 'female-producing' spermatozoa came to have the greater potency. Were this assumption correct it should receive confirmation both from the results of the experiments in which inbred females were paired with stock males and from the experiments in which stock females were paired with males from different generations of the two inbred series. The litters obtained in the former case should show a nearly equal proportion of the sexes (provided it was merely a matter of chance which kind of spermatozoa fertilized the ova), since the males were outbred and therefore, theoretically, the two kinds of spermatozoa had equal power to fertilize the ova. In the latter case the litters obtained should show a high sex ratio when the sire came from the $A$ series of inbreds and a low sex ratio when the sire belonged to the $\mathrm{B}$ series.

As shown in table 4 and in table 5, the half-inbred litters produced by the mating of inbred females with stock males gave sex ratios that were very far from equality. In only one generation of each series was there an approximately equal proportion of the sexes, in all other cases the variation was in a definite direction: in the $\mathrm{A}$ series there was an excess of males; in the $\mathrm{B}$ series the females predominated. In both series, moreover, the sex ratios in the half-inbred litters were much eloser to those in the corresponding inbred litters than they were to the norm. The uniformity in the various series of records and the small size of the 
probable error of the mean exclude the possibility that the sex ratios could have been produced by chance or by environmental action. The results, therefore, do not support the contention that the male is the chief factor in determining the sex ratio in the rat.

The sex ratio in each of the three groups of litters obtained by the mating of stock females with males from various generations of the two inbred series was below the norm, whether the sire of of the litters belonged in the $\mathrm{A}$ or the $\mathrm{B}$ series. The sex ratio in the group of litters sired by males from the A series (102.3 $\sigma^{T}$ : 100 \%) was only 6 points higher than that in the litters sired by males from the $\mathrm{B}$ series (96.2 $\sigma^{7}: 100$ \%). The results in this case, therefore, do not indicate that inbreeding, with selection, influenced the potency of the spermatozoa in any way; they seem rather to signify that the particular stock females used for breeding tended to attract spermatozoa that were 'female-producing' rather than those that were 'male-producing.'

The results of the various experiments in which inbred and outbred animals were paired, taken in connection with those from the experiments in which matings were made between litter brother and sister, seem to show that in the rat, as in Drosophila (Moenkhaus), the female has a greater influence than the male in determining the sex ratio, and that chance alone cannot be the factor that determines whether an egg shall be fertilized by a 'male-producing' or by a 'female-producing' spermatozoön.

The size of the probable error of the mean (tables 6 and 7) indicates that in each series the difference between the sex ratio for the group of inbred litters and that for the group of half-inbred litters is a significant one. Apparently, therefore, the chemotactic reaction between the ovum and the spermatozoön is not quite the same where these sexual elements come from unrelated individuals as when they are produced by individuals that are closely inbred. A somewhat analogous case is found in the hermaphroditic ascidian, Ciona, where normally, as Castle ('96) and Morgan ('04, '05) have shown, the eggs are not fertilized by spermatozoa from the same individual, although they are readily fertilized by spermatozoa from any other individual, while the 
spermatozoa from the first animal are functional when used with ova of another animal. Morgan ('14) has suggested that the infertility of the eggs of Ciona to spermatozoa from the same individual may be due to the similarity in the hereditary complex of the germ cells which in some way decreases the chances of their uniting. The selective fertilization experiments made by Marshall ('10) with different varieties of dogs and also my own experiments with different varieties of rats show that the ova of these animals have a strong tendency to unite with spermatozoa from individuals belonging to unrelated stock rather than with spermatozoa from individuals of the same 'blood.' When my own experiments are completed the results will show, I hope, whether there is a still more delicate chematactic reaction between the ova and the spermatozoa which will lead to the production of more males than females among the hybrid offspring. The anomalous sex ratios that appear in $\mathrm{F}$, hybrids almost invariably show an excess of males. This suggests that the greater the difference between individuals as regards theis blood relationship the stronger is the attraction between the ova and the "male-producing spermatozoa. If this suggestion proves true, its converse ought also be to true, and in a closely inbred line we would expect that the chemotactic reaction between the ova and the spermatozoa would be such that an excess of females would be produced. Such a possibility is not incompatible with the results of the present investigation, since in the inbred strain, as a whole, the sex ratio was below the norm, while the sex ratios in the litters of the female line (B) showed a greater deviation from the norm than did the sex ratios in the litters of the male line (A).

The results of this series of experiments, as a whole, seem to indicate that in the rat, as in the pigeon (Riddle, '14, '16, '17), in Drosophila (Moenkhaus, '11) and in the guinea-pig (Papanicolau, '15), the female has more influence in determining the sex ratio than has the male. Yet it is not in the differentiation of the ova, nor in the development of the spermatozoa, that the key to the riddle of sex-determination will be found. A knowledge of the interaction of the germ cells, and of the conditions that influence it, must be gained before the final solution of this problem can be attained. 